\title{
4
}

\section{PROGRAMA PILOTO DE PREVENCIÓN DEL ACOSO SEXUAL Y SEXISTA CENTRADO EN LOS OBSERVADORES. PROPUESTA DE INNOVACIÓN EDUCATIVA DE LA FACULTAD DE CIENCIAS \\ PILOTBYSTANDER-FOCUSEDPROGRAM TO PREVENT SEXUAL AND SEXISTHARASSMENT. TEACHING INNOVATION PROPOSAL OF THE FACULTY OF SCIENCES}

Águeda M.ํ González-Rodríguez

aglerod@ull.edu.es

Facultad de Ciencias,

Universidad de La Laguna, España

Rosaura González-Méndez

mrglez@ull.edu.es

Facultad de Psicología,

Universidad de La Laguna, España 


\section{RESUMEN}

Fruto de una colaboración interdisciplinar, la Facultad de Ciencias desarrolló, durante el curso 2017-18, un programa piloto orientado a la formación de observadores entre el alumnado del centro. El objetivo general era preparar a estudiantes para intervenir de forma temprana frente a conductas inapropiadas. Además de informar y sensibilizar sobre el acoso y sus consecuencias, las actividades han estado dirigidas a desarrollar habilidades de comunicación eficaz, resolución de conflictos y empatía. Para evaluar la eficacia de la intervención, se utizó un diseño pre-post con dos grupos (intervención y control). Se diseñó un instrumento con cuatro sub-escalaspara medir: a) la adecuación dedistintas situaciones; b) la necesidad de intervenir en cada una de ellas; c) el conocimiento sobre cómo intervenir; y d) la disposición a intervenir.El instrumento fue depurado con alumnado de otros centros, demostrandobuenas propiedades psicométricas, evidencia de validez y capacidad discriminativa. Durante las Jornadas de Innovación Educativa presentamos únicamente los resultados preliminares del estudio exploratorio ( $N=201)$, puesto que aún no contábamos con los resultados definitivos. Encontramos diferencias significtivas por género en varias medidas. Las mujeres valoraron peor las situaciones de acoso, mostraron más disposición e intención de intervenir, y mayor empatía haciaquienes sufren acoso que los varones.Los análisis de medidas repetidas mostraron mejoras significativas en el grupo de intervención en varias de las medidas (valoración del acoso sexual, conocimiento y disposición a intervenir, y empatía).

PALABRAS CLAVE:empatía; género; iguales; intervención.

\section{ABSTRACT}

As a result of an interdisciplinary collaboration, the Faculty of Sciences developed, during the academic year 2017-18, a pilot program aimed at the training of bystanders among the students of the center. The general objective was to prepare students to intervene early in the face of inappropriate behavior. In addition to informing and raising awareness about sexual harrasment and its consequences, the activities have been aimed at acquiring effective communication skills, conflict resolution and empathy. To evaluate the effectiveness of the intervention, a pre-post design with two groups (intervention and control) was used. An instrument with four sub-scales was designed to measure: a) the adequacy of different situations; b) the need to intervene in each of them; c) knowledge about how to intervene; and d) the willingness to intervene. The instrument was refined with students from other centers, demonstrating good psychometric properties, evidence of validity and discriminative capacity. During the Educational Innovation Conference we presented only the preliminary results of the exploratory study $(N=201)$, since we still did not have the final results. In addition to develop a new instrument, the results indicated significant differences by gender in several measures. Women rated harassment situations worse, showed more willingness and intention to intervene, and greater empathy towards those who suffer harassment than men do. The repeated measures analyses showed significant improvements in the intervention group in several measures (assessment of sexual harrassment, knowledge and willingness to intervene, and empathy).

KEYWORDS: empathy; gender; intervention; peers. 


\section{INRODUCCIÓN}

Según un reciente informe (NationalAcademies of Sciences, Engineeringand Medicine, 2018), el acoso es un problema prevalente en el ámbito académico, que afecta a la trayectoria académica y profesional de las mujeres. Además de consecuencias negativas sobre la salud física ypsicológica, el acoso reduce el rendimiento y la motivación de las mujeres, pudiendo llegar a provocar el abandono de la carrera académica, con laconsiguiente pérdida de talento.A partir de la revisión de los programas que han demostrado su eficacia, el citado informe recomienda a las instituciones académicas queapuesten por intervenciones que capaciten para interrumpir e intervenir cuando se esté ante un comportamiento inapropiado. Pide también que losprogramas se orienten a cambiar las conductas, no solo las creencias, y que se evalúen las intervenciones para conocer si son o no eficaces, y quéaspectos de la capacitación son más importante para cambiar la cultura de la institución. En esta línea, la Facultad de Ciencias de la Universidad de La Laguna (ULL) puso en marcha, durante el curso 2017-18, unprograma piloto para prevenir el acoso sexual y sexista.

El programase propuso formar a un grupo de estudiantes como promotores de relaciones positivasentre sus iguales. En este sentido, sigue la línea de las intervenciones dirigidas a aprovechar el potencial de los observadores como agentes de cambio dentro de las universidades (McMahon, Palmer, Banyard, Murphyy Gidycz, 2017).El objetivo general eracapacitar a dicho alumnado para intervenir de forma temprana frente a conductas inapropiadas, así como para iniciar acciones que cambien la cultura dentro del centro.De forma más concreta, las actividades perseguíanla consecución de cuatro objetivos específicos: a) aprender a discriminar entre conductas apropiadas e inapropiadas; b) saber cuándo es necesario intervenir; c) mejorar la empatía y la disposción a intervenir; y d) saber cómo actuar en cada caso. Las sesiones fueron diseñadas para favorecer la participación activa del alumnado, consiguiendo así una mayor implicación en el proceso de cambio.En paralelo a las sesiones, el alumnado dedicó parte del tiempo de trabajo autónomo a diseñar acciones de sensibilización dentro del centro. De esta forma, se pretendía que observaran la realidad del centro y tradujeran las alternativas a un lenguaje más cercano a sus iguales.

La evaluación de la eficacia de la intervención se hizo a través de un diseño pre-post, en el que se incluyó un grupo de comparación con el que no se hizo ninguna intervención. En consonancia con los objetivos específicos, desarrollamos un instrumento que nos permitiría evaluar los cambios en cada uno de ellos. El desarrollo de este instrumento se hizo a través de varias fases, con alumnado de otros centros de la ULL. Una primera fase exploratoria, en la que analizamos las propiedades psicométricas y la validez del instrumento, y una segunda fase dirigida a confirmar la estructura factorial de la escala.Durante las Jornadas de Innovación Educativa se presentaron únicamente los resultados preliminares del estudio exploratorio, ya que en ese momento aún no se contaba con los resultados del estudio confirmatorio. 


\section{ESTUDIO EXPLORATORIO}

Una revisión de los estudios sobre el acoso en las universidades, nos llevó a comprobar que existían algunos instrumentos en inglés y otros en español sin validar. No obstante, ninguno se ajustaba a la necesidad de evaluar cambios en los cuatro objetivos planteados. Algunos de estos instrumentos sirvieron de base, no obstante, para desarrollar un conjunto inicial de ítems con los que realizar un estudio exploratorio.

Los mismos ítems fueron evaluados a través de cuatro sub-escalas que respondían a las siguientes cuestiones: a) en qué medida consideraban adecuadas o inadecuadas las distintas situaciones; b) si consideraban adecuado intervenir; c) si sabrían cómo intervenir; y d) su disposición a intervenir.

En este primer estudio participaron 201 estudiantes de distintos grados que no pertenecen a la Facultad de Ciencias (72\% eran mujeres). Tras los análisis factoriales exploratorios, extrajimos único factor para cada sub-escala, con los mismos once ítems. La tabla 1 muestra los valores de KMO, la varianza explicada y la consistencia interna de los ítems en cada una de las medidas.

\begin{tabular}{|cccc|}
\hline \multicolumn{4}{|c|}{ TABLA 1: Resultados Del ANÁlISIS FACTORIAL EXPLORATORIO CON CADA SUB-ESCALA } \\
\hline Medidas & KMO & \% Varianza explicada & $\alpha$ \\
\hline Valoración & .890 & 58.3 & .805 \\
\hline Necesidad de intervenir & .938 & 65.7 & .933 \\
\hline Sabe cómo intervenir & .898 & 45.9 & .880 \\
\hline Disposición a intervenir & .853 & 41.9 & .847 \\
\hline
\end{tabular}

Por otro lado, la evidencia de validez se obtuvo comparando nuestrassubescalas con otras que miden constructos similares. Concretamente, utilizamos tres escalas de Edwards et al. (2017), que miden empatía hacia la persona que sufre acoso $(\alpha=.80)$, intención de intervenir $(\alpha=.73)$ y negación del problema $(\alpha=.70)$. Aunque no encontramos relación entre la empatía y los factores de nuestra escala, se confirmó que las sub-escalas correlacionaban positivamente con la disposición a intervenir y negativamente con la negación del problema.

\section{VALIDEZ DISCRIMINANTE}

La evidencia de validez discriminante se obtuvo comparando los resultados en cada una de las cuatro sub-escalas por género. Tras comprobar la homogeneidad de las varianzas, un análisis univariado (ANOVA) mostró diferencias significativas en dos de nuestras medidas (valoración de las situaciones y disposición a intervenir) y en dos de las medidas adicionales (empatía e intención de intervenir). Tal como puede verse en la tabla 2, los varones mostraron mayor aceptación de los indicadores de acoso y menor disposición a intervenir. En cuanto a las medidas adicionales (Edwardset al., 2017), las estudiantes mostraron mayor empatía e intención de intervenir. 
TABLA 2: Resultados del ANOVA COMPARANDO LAS RESPUESTAS DE VARONES Y MUJeRES

\begin{tabular}{|cccccccc|}
\hline Sub-escalas & M varones & DT & M mujeres & DT & $\mathrm{F}(1,199)$ & $\mathrm{p}$ & $\mu \mathrm{p} 2$ \\
\hline Valoración & .40 & 1.16 & -.15 & .88 & $10.07^{*}$ & .002 & .033 \\
\hline Necesidad de intervenir & -.17 & .83 & .06 & 1.05 & 2.37 & $\mathrm{~ns}$ & - \\
\hline Sabe cómo intervenir & .24 & .92 & -.08 & 1.01 & 2.79 & $\mathrm{~ns}$ & - \\
\hline Disposición a intervenir & -.27 & 1.05 & .10 & .96 & 4.41 & .038 & .028 \\
\hline Medidas adicionales & & & & & & & \\
\hline Empatía & -.29 & 1.02 & .11 & .97 & 6.91 & .009 & .049 \\
\hline Intención de intervenir & -.48 & 1.05 & .18 & .92 & 19.31 & .000 & .058 \\
\hline Negación del problema & .11 & 1.01 & -.04 & .99 & 1.06 & $\mathrm{~ns}$ & - \\
\hline
\end{tabular}

Nota: * en este caso se utilizó el estadístico robusto de Welch $(1,78.57)$.

\section{EvaluaCión DE LA INTERVENCIÓN}

Como ya hemos indicado, la evaluación de la intervención se hizo a través de una evaluación pre-post. En el grupo de intervención participaron 30 estudiantes de distintos grados de la Facultad de Ciencias (contamos con un grupo de control de similares características). Los análisis de medidas repetidas realizados antes y después de la intervención mostraron mejoras significativas en tres de las sub-escalas (véase la Tabla 3). Tras la intervención, el alumnado valoró más negativamente los indicadores de acoso, indicó más disposición a intervenir y más conocimiento de cómo hacerlo. De igual forma, mejoró la medida de empatía. Como esperábamos, no encontramos diferencias significativas en el grupo de control.

\begin{tabular}{|ccccccccc|}
\hline \multicolumn{7}{|c|}{ TABLA 3: ReSULTADOS DEL ANÁLISIS MEDIDAS REPETIDAS CON EL GRUPO DE INTERVENCIÓN } & \\
\hline Sub-escalas & M Pre & DT & MPost & DT & $\begin{array}{c}\text { F } \\
(1.29)\end{array}$ & $\mathrm{p}$ & $\mu \mathrm{p} 2$ \\
\hline Valoración & 1.28 & .17 & 1.16 & .17 & 5.14 & .031 & .150 \\
\hline Necesidad de intervenir & 3.60 & .57 & 3.51 & .88 & .12 & $\mathrm{~ns}$ & - \\
\hline Sabe cómo intervenir & 2.66 & .57 & 3.39 & .70 & 17.89 & .000 & .381 \\
\hline Disposición a intervenir & 3.13 & .32 & 3.49 & .58 & 7.37 & .011 & .203 \\
\hline Medidas adicionales & & & & & & & \\
\hline Empatía & .74 & .29 & .92 & 1.71 & 7.82 & .009 & .212 \\
\hline Intención de intervenir & 2.83 & .33 & 3.04 & .45 & 4.13 & $\mathrm{~ns}$ & - \\
\hline Negación del problema & .33 & .09 & .34 & .13 & .279 & $\mathrm{~ns}$ & - \\
\hline
\end{tabular}




\section{CONCLUSIONES}

En estos momentos, contamos ya con un instrumento validado que permite evaluar este tipo de intervenciones. Se trata de un instrumento que ha demostrado tener buenas propiedades psicométricas, evidencia de validez y capacidad discriminativa.

Las diferencias por género detectadas en algunas de las sub-escalas subrayan la necesidad de hacer prevención con el alumnado. Los varones tienden a normalizar situaciones que las mujeres consideran acoso.

Durante la evaluación del proceso, el alumnado que participó en la intervención mostró satisfacción con el desarrollo de la misma. Entre las propuestas de mejora, se subrayó la necesidad de aumentar el tiempo dedicado a resolver supuestos concretos para tener mayor seguridad a la hora de intervenir.

El programa piloto mostró mejoras significativas en el grupo de intervención en todas las sub-escalas, excepto la necesidad percibida de intervenir, donde se produjo un efecto techo. De igual forma, se detectó un aumento de la empatía hacia las personas que sufren acoso.

En lugar de esperar a que la denuncia de acoso se produzca, el programa ofrece una alternativa desde la prevención, fomentando la intervención temprana entre iguales y el cambio de cultura dentro del centro.

\section{REFERENCIAS BIBLIOGRÁFICAS}

Edwards, K. M., Banyard, V. L., Sessarego, S. N., Stanley, L. R., Kimberly, J., Eckstein, R. P. y LEYVA, P. C. (2017). Measurement tools to assess relationship abuse and sexual assault prevention program effectiveness among youth. Psychology of Violence, 8(5), 537-545. doi: 10.1037/vio0000151.

National Academies of Sciences, Engineeringand Medicine (2018). Sexual Harassment of Women: Climate, Culture, and Consequences in Academic Sciences, Engineering, and Medicine. Washington, DC: The National Academies Press. doi: $10.17226 / 24994$.

McMahon, S., Palmer, J. E., Banyard, V., Murphy, M. y Gidycz, C. A. (2017). Measuring bystander behavior in the context of sexual violence prevention: Lessons learned and new directions. Journal of Interpersonal Violence, 32, 2396-2418. doi: $10.1177 / 0886260515591979$. 